\title{
Micro-grasping Forceps Manipulator for MR-Guided Neurosurgery
}

\author{
Nobuhiko Miyata ${ }^{1}$, Etsuko Kobayashi ${ }^{1}$, Daeyoung Kim ${ }^{1}$, Ken Masamune ${ }^{5}$, \\ Ichiro Sakuma ${ }^{1}$, Naoki Yahagi $^{1}$, Takayuki Tsuji ${ }^{1}$, Hiroshi Inada ${ }^{2}$, \\ Takeyoshi Dohi ${ }^{3}$, Hiroshi Iseki ${ }^{4}$, and Kintomo Takakura ${ }^{4}$ \\ ${ }^{1}$ Graduate School of Frontier Scineces, the University of Tokyo, \\ 7-3-1Hongo Bunkyo-ku Tokyo, 113-8656, Japan \\ ${ }^{2}$ Graduate school of Information, the University of Tokyo, \\ ${ }^{3}$ Graduate School of Engineering, the University of Tokyo, \\ ${ }^{4}$ Department of Neurosurgery, Tokyo Women's Medical University, \\ ${ }^{5}$ Collage of Science \& Engineering, Tokyo Denki University
}

\begin{abstract}
Mechanical support system is needed for minimally invasive surgery, since it enables precise manipulation of surgical instruments beyond human ability in a small operation space. Furthermore, a robot available for intraoperative MRI guided neurosurgical procedures could allow less invasive and more accurate image guided surgery. By combination of precise positioning to the target by intra-operative MRI guided surgery and dexterity by the multi function micromanipulator, safe and smooth operation is expected to be performed. In this approach, we have developed MR-compatible micro-forceps manipulator of the multi-function micromanipulator system for neurosurgery. By a new cam mechanism for two degrees of bending freedom, we achieved these excellent characteristics for the micro forceps. 1) Simple mechanism suitable for a micromanipulator, 2) Precise positioning, 3) Suitable mechanism for MR compatible manipulator. By evaluation experiments, we confirmed precise positioning of the manipulator and MR compatibility of the manipulator.
\end{abstract}

\section{Introduction}

Minimally invasive surgery is currently a hot topic because it can reduce a patient's post-operative discomfort and length of rehabilitation period, thus improving the patient's Quality of Life. Minimally invasive surgery has many advantages to the patient; on the contrary, it reduces the surgeon's dexterity. Precise manipulation of surgical forceps is indispensable for safe surgery, however, in minimally invasive neurosurgery, the precise manipulation of forceps through a small burr hole is particularly difficult. This difficulty is caused by, for example, hand trebling and insufficient degrees of freedom (D.O.F.) of the forceps. This has restricted the number of applicable clinical cases.

To achieve precise manipulation and enhance the surgeon's dexterity, mechanical operation support is useful. In this approach, various kinds of such surgical support systems have been developed [1][2]. In the neurosurgery, also, a mulch function mi- 
cromanipulator system has been reported [3]. We have also developed a multifunction micromanipulator system (Fig.1) [4]. It consists of two micro grasping forceps manipulators $(\phi 3.2 \mathrm{~mm})$, a rigid neuro-endoscope $(\phi 4 \mathrm{~mm})$, a suction tube $(\phi 3 \mathrm{~mm})$, and an irrigation tube $(\phi 1.4 \mathrm{~mm})$. The components are inserted into a rigid tube $(\phi 10 \mathrm{~mm})$. The micromanipulator is inserted into the burr hole with an insertion manipulator [5] that can determine the direction and position of the manipulator by reference to pre-operative images such as CT and Magnetic Resonance Imaging (MRI). The micro grasping forceps manipulator system has two degrees of bending freedom by a wire and a ball joint mechanism. However, the problem of the manipulator was stick-slip caused by wire mechanism and friction.

MRI techniques, including MRI angiography and functional MRI, are attractive for the development of interventional MRI therapies and operations [6][7][8]. A robot available for these neurosurgical procedures could allow less invasive and more accurate image guided surgery. From this background, we have developed MR-compatible stereo-tactic insertion manipulator [9].

From these backgrounds, by combination of precise positioning to the target by intra-operative MRI guided surgery and dexterity by the multi-function micromanipulator, safe, certain and smooth operation is expected to be performed.

Therefore, the goal of this research is achieve a micro grasping forceps manipulator of the multi-function micromanipulator. And the system has these characteristics; 1) two degrees of bending freedom, 2) precise positioning, and 3) MR-compatibility.

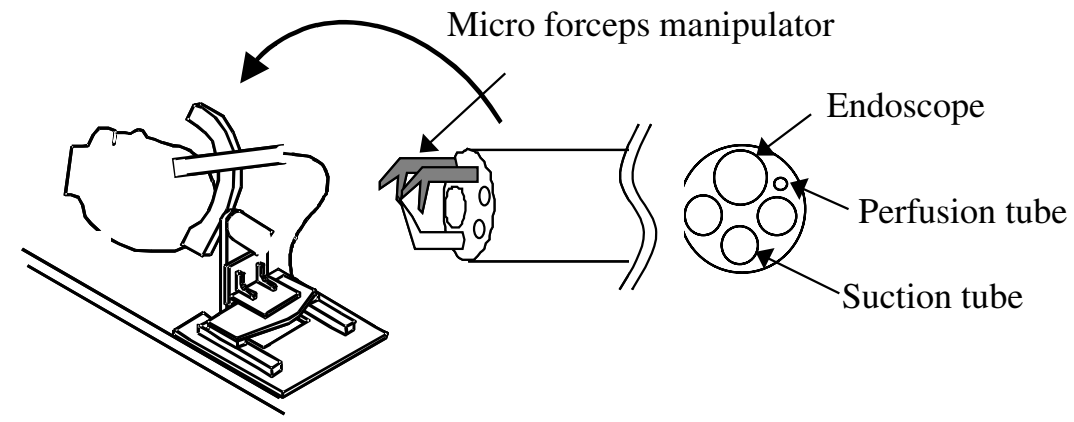

Fig. 1. Multi-function micromanipulator system for neurosurgery

\section{Micro-grasping Forceps Manipulator}

\subsection{Requirements}

Requirements of the micro grasping forceps are as follows;

1) MR-compatibility

2) Size; $3 \mathrm{~mm}$ in diameter. As shows in Figure 1, the micro grasping forceps is inserted into a $10 \mathrm{~mm}$ rigid tube. The size must be small.

3) Moving degrees of freedom (D.O.F.): It must have at least five D.O.F. Two degrees of bending, rotation around the axis, back and forth movement and grasping. 
4) Precision positioning; Because the target is small tissue, the precise positioning is required.

Furthermore, because the manipulator requires small size with multi degrees of freedom, its mechanism must be simple.

\subsection{MR Compatibility}

MR-compatible material is the material that does not effect MR images and is not effected from strong magnetic field [10]. We validated MR-compatibility of materials and selected adequate material for the manipulator. $\phi 3 \mathrm{~mm}$ shaft was inserted into the beaker of $107 \mathrm{~mm}$ in diameter and measured the range of artifact caused by the shaft.

Figure 2 shows the MR image and the range of artifact. We used MRI with 0.5T static magnetic field (MRH-500 Hitachi Medical corp., Japan) and the protocols of the image were T1 enhanced image, spin echo, 256x256mm FOV, 1mm image resolution, TR/TE $1500 / 35$ and $2.0 \mathrm{~mm}$ slice thickness. The image was taken in coronal plane. The range of the artifact caused by a stainless steel was almost same with a circle of $60 \mathrm{~mm}$ in diameter. Aluminum and titan effected about the range of the circle of $15 \mathrm{~mm}$ in diameter. Therefore, we used mainly titan and aluminum for the micro grasping forceps and used stainless steel for the part set far from the target.

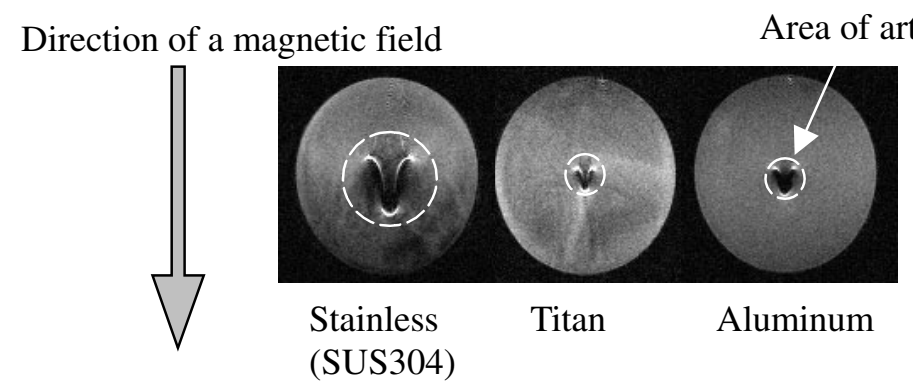

Fig. 2. MR image of the water with shafts of $3 \mathrm{~mm}$ in diameter

\subsection{New Cam Mechanism for a Micro-grasping Forceps Manipulator}

To realize these requirements such as small size and precision positioning, we proposed a new cam mechanism for two degrees of bending freedom (Fig.3). A torsion spring is attached to a grasping forceps part and a cylinder cut aslant (named a cam cylinder) is set around the spring. The base of the grasping forceps part is push to an outer cylinder around the cam cylinder. Then by pushing the base of the grasping forceps part by the cam cylinder, the forceps manipulator is bend and by rotating the cam cylinder, bending direction can be changed. Rotation of the forceps is determined by the rotating the spring. Back and forth movement is achieved by a rack and pinion and grasping is by a wire. As we mentioned in chpt.2.2 we mainly used Aluminum and Titan for the manipulator. For the spring, we used $\mathrm{Be}-\mathrm{Cu}$. Ultrasonic motors drive all mechanisms. 
Figure 4 shows a tip of the micro forceps manipulator. The diameter of the forceps was $3.2 \mathrm{~mm}$. Figure 5 shows overview of the multi-function micromanipulator system.

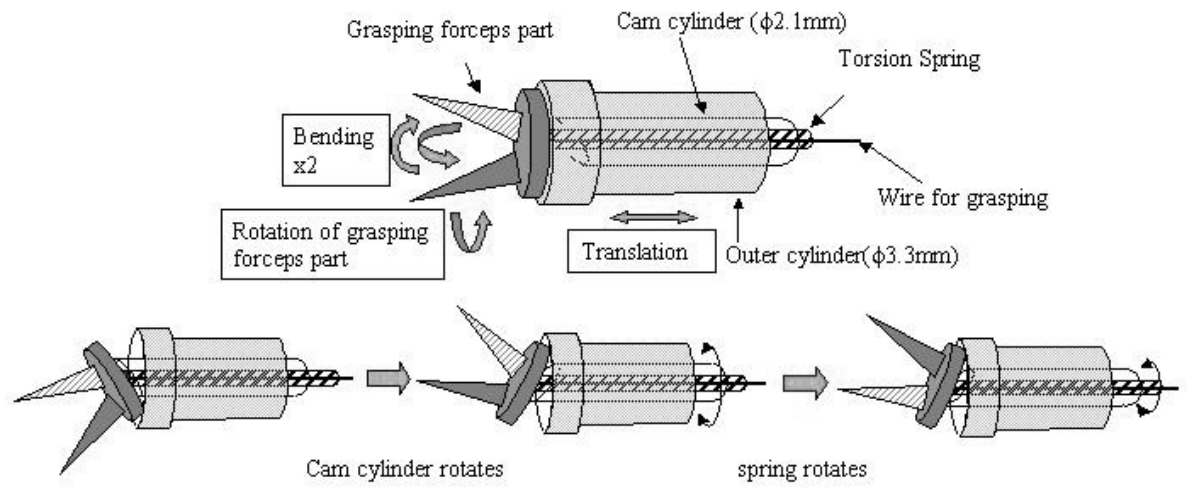

Fig. 3. Mechanism of the micro grasping forceps

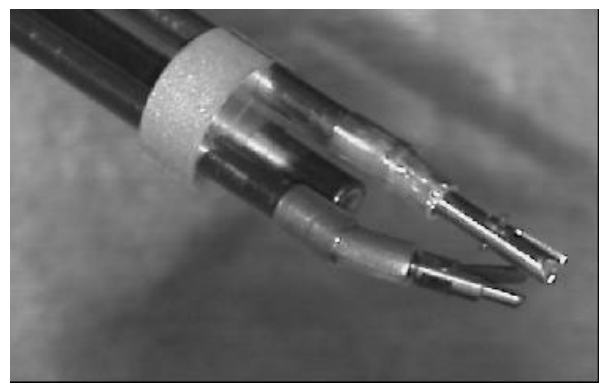

Fig. 4. Micro grasping forceps manipulator

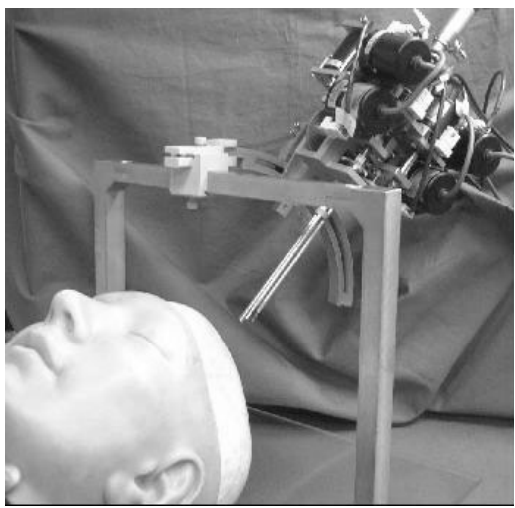

Fig. 5. Overview of the multi-function micromanipulator system

\section{Experiment}

\subsection{Positioning Accuracy}

We evaluated the positioning accuracy of the bending when we put the command to move 1.0 degree. We set the high-definition digital micro scope (VH-6300, Keyence co. ltd., Japan) to observe the bending angle and measured the bending angle using the microscopic image (image resolution $0.015[\mathrm{~mm} / \mathrm{pixel}]$ ).

Figure 6 shows the relationship between the pushing distance of the cam cylinder and the measured bending angle. Average of the bending angle was 0.98 degree and standard deviation of the error was 0.68 degree. In bending movement, we achieved precise positioning without stick-slip movement. However, when the bending angle was more than 30 degree, the error was big, because the spring was extended rather than bending. 


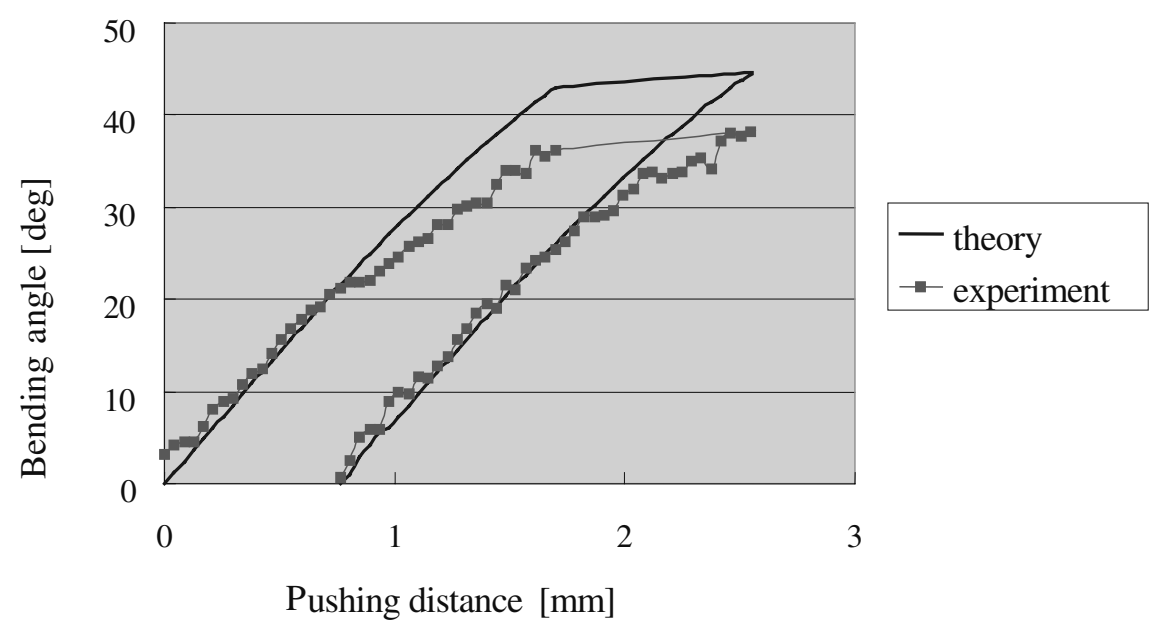

Fig. 6. Positioning accuracy of the bending

\subsection{Evaluation of MR Image}

We performed an evaluation of the MR image distortion. We put a sphere of $63 \mathrm{~mm}$ in diameter into water and put the forceps on the surface of the sphere. We used a same MRI devices with the MR-compatible material evaluation (0.5T static magnetic field, MRH-500 Hitachi Medical corp.) The protocols of the image were T2 enhanced image, spin echo, 256x256mm FOV, 1mm image resolution, TR/TE 2500/100 and $2.0 \mathrm{~mm}$ slice thickness. The image was taken in sagittal plane. For contrast, the non MR-compatible forceps manipulator was evaluated.

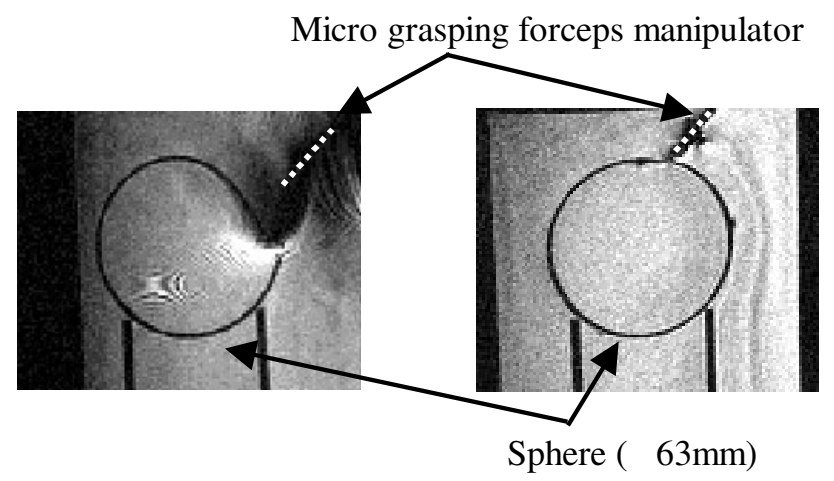

Fig. 7. MR Image of a sphere with the micro forceps manipulator. Left: Image with non MRcompatible forceps. Right: Image with MR-compatible forceps

Figure 7 shows MR Images of the $\phi 63 \mathrm{~mm}$ sphere with the non MR-compatible and MR-compatible micro forceps manipulator. We measured the radius of the sphere from both images. In non MR-compatible forceps, maximum error of the radius was 
$5.3 \mathrm{~mm}$. On the contrary, maximum error of the MR-compatible forceps was $1.5 \mathrm{~mm}$. From this result, the micro grasping forceps manipulator has enough MR compatibility to be used for intra-operative MRI guide surgery.

\section{Discussion}

Because the cam mechanism we have developed is simple and it has certain transmission of the positioning compare to wire mechanism, the manipulator has excellent characteristics as the micro forceps manipulator.

1) It consists of only simple parts. Therefore manufacturing and assembling is easily completed even if small parts are required.

2) Compare to the wire mechanism, precise positioning is possible.

3) It is also suitable for MR compatible manipulator.

In the experiment, we confirmed the precise positioning of $0.68 \mathrm{~mm}$ error.

In the image distortion evaluation, we confirmed little image distortion (maximum $1.5 \mathrm{~mm}$ ) caused by the manipulator. Because image resolution was $1.0 \mathrm{~mm}$, the distortion was small enough to the positioning.

For MR-compatible materials, we used $\mathrm{Be}-\mathrm{Cu}$ spring. The spring constant was $12.6 \mathrm{gf} / \mathrm{mm}$ and it was not enough strength for the mechanism. We have to reselect the MR-compatible spring for more precise positioning and high torque occurrence.

Kan [3] achieved neurosurgery assist robotic system (HUMAN). In this system, however, forceps manipulator has only one degree of bending freedom. For dexterous operation, it is preferable to have the same degrees of freedom with the human wrist for the movement of the forceps. From this point of view, we have developed the forceps with two degrees of bending freedom and our system provide sufficient dexterity.

\section{Conclusion}

We have developed MR-compatible forceps manipulator for the multi-function micromanipulator system. For the bending mechanism we proposed new simple cam mechanism. By this forceps manipulator, we achieved precise bending positioning (0.68 degree) and MR compatibility (Maximum $1.5 \mathrm{~mm}$ image distortion).

For future work, we combine the forceps and the MR-compatible insertion manipulator [9], and image distortion evaluation and positioning accuracy evaluation is performed.

\section{Reference}

1. Salisbury J. K.: The heart of microsurgery, Mechanical Engineering, v.120 n.12 Dec p4651 (1998)

2. Mitsuishi M. et al, :A tele-micro-surgery system with co-located view and operation points and a rotational-foce-feedback-free master manipulator, Proc. of MRCAS '95, pp.111-118, (1995) 
3. Berkelman P. J. et al :A Miniture instrument tip force sensor for robot/human cooperative microsurgical manipulator with enhanced force feedback, MICCAI2000, Proc. pp. 897-906 (2000)

4. Schurr M.O. et al.: Robotics and telemanipulation technologies for endoscopic surgery, Surgical endoscopy, 14, pp.375-381, (2000)

5. Kan K., et al. : Microscopic-manipulator system for minimally invasive neurosurgery, Proc. of CARS 98, pp.594-598, (1998)

6. Harada K. et al.: Development of a micro manipulator for minimally invasive neurosurgery, Proc. of CARS 2000, pp.116-120, (2000)

7. K Masamune, M Sonderegger, H Iseki, K Takakura, M Suzuki, T Dohi, Robots for Stereotactic neurosurgery, Advanced Robotics, Vol.10, No.3, pp.391-401 (1996)

8. Hwa-shain Y, et al.: Implantation of intracerebral depth delectrodes for monitoring seizures using the Pelorus stereotactic system guided by magnetic resonance imaging, J Neurosurg 78:138-141, (1993)

9. Robert B.Lufkin: Interventional MRI, pp.55-69, Mosby, (1999)

10. Chinzei K., Hata N., et al.: MR-compatible surgical assist robot: system integration and preliminary feasibility Study, IEEE/RSJ IROS2000, Proc. 727-732 (2000)

11. Masamune K., Kobayashi E., et al.: Development of an MRI compatible needle insertion manipulator for stereotactic neurosurgery, J Image Guided Surgery, 1 pp.242-248 (1995)

12. Chinzei K., et al.: Surgical assist robot for the active navigation in the intraoperative MRI, MICCAI2000, Proc. pp.921-930 2000 